\title{
Determinants of Taste Preference and Acceptability: Quality versus Hedonics
}

\author{
Gregory C. Loney, Ginger D. Blonde, Lisa A. Eckel, and Alan C. Spector \\ Department of Psychology and Program in Neuroscience, Florida State University, Tallahassee, Florida 32311
}

Several methods exist for reliably determining the motivational valence of a taste stimulus in animals, but few to determine its perceptual quality independent of its apparent affective properties. Individual differences in taste preference and acceptability could result from variance in the perceptual qualities of the stimulus leading to different hedonic evaluations. Alternatively, taste perception might be identical across subjects, but the processing of the sensory signals in reward circuits could differ. Using an operant-based taste cue discrimination/generalization task involving a gustometer, we trained male Long-Evans rats to report the degree to which a test stimulus resembled the taste quality of either sucrose or quinine regardless of its intensity. The rats, grouped by a characteristic bimodal phenotypic difference in their preference for sucralose, treated this artificial sweetener as qualitatively different-compared to sucraloseavoiding rats, the sucralose-preferring rats found the stimulus much more perceptually similar to sucrose. Although the possibility that stimulus palatability may have served as a discriminative cue cannot entirely be ruled out, the profile of results suggests otherwise. Subsequent brief-access licking tests revealed that affective licking responses of the same sucralose-avoiding and -preferring rats differed across concentration in a manner approximately similar to that found in the stimulus generalization task. Thus, the perceived taste quality of sucralose alone may be sufficient to drive the observed behavioral avoidance of the compound. By virtue of its potential ability to dissociate the sensory and motivational consequences of a given experimental manipulation on taste-related behavior, this approach could be interpretively valuable.

\section{Introduction}

Gustatory afferents and their relays project to a number of distinct brain structures, resulting in complex contributions to various behavioral and physiological outputs (for review, see Spector and Glendinning, 2009). Many taste compounds have rewarding or aversive characteristics in addition to their perceptual qualitative features. The processes underlying these sensory and hedonic properties are thought to some extent to involve different neural circuits. Although there are many behavioral testing paradigms used with animal models capable of discerning the motivational valence of a taste stimulus (i.e., palatability), it is more challenging to infer the perceptual features of a chemical compound independent of its hedonic characteristics. Arguably, the ability to dissociate between the sensory and motivational consequences of a given neural, pharmacological, or genetic manipulation on taste-related behavior is essential for a full understanding of the functional organization of the gustatory system and the general circuitry underlying reward. If one animal prefers a stimulus and another avoids it, it may be that the compound has different perceptual qualities to the respective tasters, leading to

Received Dec. 1, 2011; revised May 28, 2012; accepted June 2, 2012.

Author contributions: G.C.L., G.D.B., L.A.E., and A.C.S. designed research; G.C.L. performed research; G.C.L., G.D.B., and A.C.S. analyzed data; G.C.L., G.D.B., L.A.E., and A.C.S. wrote the paper.

This work was supported by NIH Grant T32 DC-000044 to G.C.L.

The authors declare no financial conflicts of interest.

Correspondence should be addressed to Dr. Alan C. Spector, Department of Psychology, Florida State University, 1107 West Call Street, P.0. Box 3064301, Tallahassee FL 32306-4301. E-mail: spector@psy.fsu.edu.

DOI:10.1523/JNEUROSCI.6036-11.2012

Copyright $\odot 2012$ the authors $\quad 0270-6474 / 12 / 3210086-07 \$ 15.00 / 0$ different hedonic evaluations. Alternatively, it may be that the perceived taste quality is identical, but the way the signals are processed by putative reward circuits differs.

Accordingly, whether the reported phenotypic variation in the preference for artificial sweeteners is due to differences in the processing of taste quality or reward remains to be elucidated (Sclafani and Clare, 2004; Inoue et al., 2007; Carroll et al., 2008; Loney et al., 2011). Of particular relevance to the present report, $\sim 70 \%$ of outbred rats, including Long-Evans and SpragueDawley strains, display a clear and robust rejection of sucralose and are referred to as sucralose-avoiding (SA); the remaining rats prefer sucralose over water at these same concentrations and are referred to as sucralose-preferring (SP) (Sclafani and Clare, 2004; Loney et al., 2011). Because artificial sweeteners are reported by humans to have both sweet and bitter qualities that depend on the concentration (Schiffman et al., 1995; Horne et al., 2002), if the relative strength of those components varies between animals, then the gustatory signal being processed by reward circuits may not necessarily be the same and therefore could be the basis of the observed phenotypic variation.

Here we used a novel approach, inspired by the earlier work of Morrison (1967) and a recent modified application of the technique in our laboratory (Grobe and Spector, 2008), to psychophysically address whether the phenotypic difference in the palatability of sucralose can be explained by differences in the perceived quality of the stimulus. Specifically, we used taste as a discriminative cue to guide operant responding. Rats were first categorized as SP or SA and then trained, in a custom gustometer, to respond one way if the sampled stimulus was quinine and 
another way if it was sucrose. Thus, we trained animals to report how much a given stimulus (such as sucralose), presented on test probe trials, qualitatively tasted like sucrose or quinine, without relying on the hedonic characteristics of the stimuli to drive responding.

\section{Materials and Methods Experiment 1 \\ Subjects}

Naive adult male Long-Evans rats (Charles River) were classified as SA $(n=5)$ or SP $(n=4)$ using a previously reported $24 \mathrm{~h}$ two-bottle preference protocol (Loney et al., 2011). All rats were individually housed in polycarbonate cages in a temperature- and humidity-controlled room with a 12 h light cycle (lights on 0700-1900). Each rat had ad libitum access to pelleted lab chow (Purina 5001) in its home cage. Training and testing sessions were conducted Monday through Friday. Water was removed $15-20 \mathrm{~h}$ before the first session and was returned after completion of the last session of a given week. All procedures were approved by the Florida State University Animal Care and Use Committee.

\section{Stimuli}

All training and test solutions were prepared daily with deionized reverse osmosis (RO) water, reagent grade chemicals (Sigma Aldrich), and commercial grade sucrose (Publix). All stimuli were presented at room temperature. Training stimuli consisted of 30,100 , and $300 \mathrm{~mm}$ sucrose and $0.1,0.3$, and $1.0 \mathrm{~mm}$ quinine hydrochloride (quinine). During discrimination training, it was clear that animals performed substantially worse on the lowest sucrose solution, so a decision was made to double each sucrose concentration $(60,200,600 \mathrm{~mm})$, which successfully improved performance; these sucrose concentrations, along with those listed above for quinine, are referred to hereafter as the standard stimuli. Test stimuli consisted of $0.0025,0.025,0.25,2.5,5.0,10,20,40$, and $80 \mathrm{~mm}$ sucralose (Tate and Lyle); $100 \mathrm{~mm} \mathrm{NaCl}$; artificial saliva (15 mm NaCl, $22 \mathrm{~mm} \mathrm{KCl,}$ $3 \mathrm{~mm} \mathrm{CaCl}_{2}, 0.6 \mathrm{~mm} \mathrm{MgCl}_{2}$ ); four sucrose base solutions (75, 150, 300, $600 \mathrm{~mm})$ adulterated with each of four quinine concentrations $(0.1,0.3$, 1.0, 3.0 mM); and Dasani bottled water (Coca-Cola).

\section{Training}

Spout training. For a detailed description of the testing apparatus, see Spector et al. (1990) and Grobe and Spector (2008). Rats were initially trained to lick a dry sample stimulus delivery spout centrally located through a slot in the side wall of the test chamber. After two licks, in which the interlick interval was $\leq 250 \mathrm{~ms}$, a predetermined aliquot of fluid from one of several reservoirs was deposited in the sample spout. Once the spout was filled, rats could elicit up to five more licks (receiving $\sim 5 \mu$ of fluid per lick) during the $3 \mathrm{~s}$ sample period before the sample delivery spout rotated out of place and was rinsed with water. Rats were given access to only the central sample delivery spout, the left reinforcement spout, or the right reinforcement spout for a given session, all of which provided water. This served to familiarize the rats with the opportunity to obtain fluid from each spout. All rats had access to the same spout on any given day and training lasted for $3 \mathrm{~d}, 1 \mathrm{~d}$ per spout. The duration of these training sessions and all others to follow was $40 \mathrm{~min}$.

Side training. In a counterbalanced fashion, rats were trained to associate a given reinforcement spout with a standard stimulus (sucrose or quinine) so that one stimulus was assigned to the left reinforcement spout and the other stimulus was assigned to the right reinforcement spout. Only one standard stimulus was presented per rat per day and only the highest concentration of each training compound was used $(300 \mathrm{~mm}$ sucrose or $1.0 \mathrm{~mm}$ quinine). The stimulus used was alternated for every rat, and animals were tested on the subsequent day with the other stimulus. Following stimulus sampling, each rat was given $180 \mathrm{~s}$ (limited hold) to make a response on their assigned reinforcement spout. The unused spout was removed from the cage for that particular training day. If the rat responded on the reinforcement spout within the limited hold, that response was reinforced with 20 licks (or 10 s access, whichever came first) of water. The reinforcement volume and duration were kept constant across every stage of training and testing. Regardless of the outcome (response or no response), there was a $6 \mathrm{~s}$ intertrial interval. Side training lasted a total of $4 \mathrm{~d}$, consisting of $2 \mathrm{~d}$ of training for each stimulus/spout assignment.

Alternation. Both the left and right reinforcement spouts were available during this phase of training. Alternation training sessions consisted of presentation of one of two standard stimuli $(300 \mathrm{~mm}$ sucrose or $1.0 \mathrm{~mm}$ quinine) repeatedly until a criterion number of correct responses occurred (6, 4, or 2 on training days 1,2 , and 3 , respectively), after which the other stimulus was presented and the animal was required to reach the same criterion before the original stimulus was returned. The correct responses did not have to occur sequentially. The limited hold for alternation training was reduced from 180 to $15 \mathrm{~s}$ and a $10 \mathrm{~s}$ timeout was delivered in the case of an incorrect response or failure to make a response.

Discrimination training I-III. For stage I of discrimination training, the intermediate concentration of each standard stimulus (100 mm sucrose and $0.3 \mathrm{~mm}$ quinine) was presented in randomized blocks. Animals were required to make a choice about the taste quality of the sample stimulus by responding on one of the two reinforcement spouts. Rats were given $15 \mathrm{~s}$ to respond after sampling and incorrect responses or no responses were punished with a $10 \mathrm{~s}$ timeout. After reaching a set criterion $(80 \%$ correct for each stimulus), animals moved on to stage II.

During stage II of discrimination training, all three concentrations of both quinine and sucrose were presented in blocks of six randomized trials (without replacement). The timeout for an incorrect response was increased to $20 \mathrm{~s}$. Because animals had reached $80 \%$ correct responses for all stimuli but the lowest sucrose concentration, all rats were advanced to stage III of discrimination training and each of the three sucrose concentrations was doubled.

During stage III, a partial reinforcement schedule was instituted such that $\sim 15 \%$ of the total trials were unreinforced in the case of a correct response. This was done in anticipation of the testing paradigm in which presentation of a test stimulus would not be reinforced or punished. The duration of the limited hold for stage III was reduced to $5 \mathrm{~s}$. The timeout for responses on the incorrect reinforcement spout was removed but remained in the instance where a rat did not respond by the end of the limited hold; the timeout duration was increased to $30 \mathrm{~s}$. This was done to promote the initiation of a larger number of trials within the $40 \mathrm{~min}$ testing session and because the animals were responding at very high levels of performance to the standards. Animals were run in stage III until it was determined that the partial reinforcement schedule and the removal of the punishment for incorrect responses did not interfere with the ability of each rat to perform at or above $80 \%$ correct responding overall. Stage III discrimination training parameters served as a training refresher on the Monday sessions during testing. There was a total of 65 training sessions across all phases for each rat.

\section{Testing}

The testing phase modeled stage III discrimination training though the unreinforced test stimuli were introduced on Tuesday-Friday of each week; these test stimulus trials constituted $\sim 15 \%$ of the total trials per test day in addition to the reinforced sucrose and quinine standards. During a given 4-d-testing week, four separate concentrations of sucralose ( presented in ascending order) were presented one per day and then that week of testing was repeated the following week before moving to the next block of sucralose concentrations so that each concentration was tested twice on noncontiguous days. As we had an uneven number of sucralose concentrations, $80 \mathrm{~mm}$ sucralose was tested in a block with RO water. On the second week of sucralose testing of the lowest four concentrations, the order of testing for $0.0025 \mathrm{~mm}$ and $0.025 \mathrm{~mm}$ concentrations was unintentionally switched. The sessions with $100 \mathrm{~mm} \mathrm{NaCl}$, Dasani water, and artificial saliva serving as test stimuli followed the phase of testing with the sucralose concentrations. These testing sessions emulated sucralose testing with each of the three stimuli $(100 \mathrm{~mm} \mathrm{NaCl}$, Dasani water, and artificial saliva in order of testing) presented on a given day and that same block repeated the following week. After those weeks of testing, each of the four sucrose base solutions were tested in separate weeks $(600,300,150,75 \mathrm{~mm}$ in order of testing), with each of four quinine adulterations constituting one of the $4 \mathrm{~d}$ within a week (tested in ascending order). 


\section{Water control sessions}

To ensure that animals were responding solely to the chemical properties of the training stimuli, two water control sessions were conducted in which each reservoir was filled with RO water and arbitrarily assigned as one of the three concentrations of each standard stimulus. There were 53 total test sessions across all phases for each rat.

\section{Data analysis}

A Sucrose Generalization Score (Grobe and Spector, 2008) for each test stimulus was calculated for each rat on the basis of the performance to the highest concentrations of the standard stimuli, effectively quantifying the degree to which the animals reported that the taste quality of the test stimulus resembled sucrose:

$$
\text { Sucrose Generalization Score }=\frac{P(T)-(1-P(Q))}{P(S)-(1-(P(Q))}
$$

where $P(Q)$ represents the proportion of correct responses to the highest $(1.0 \mathrm{~mm})$ quinine concentration, $P(S)$ represents the proportion of correct responses to the highest $(600 \mathrm{~mm})$ sucrose concentration, and $P(T)$ represents the proportion of times the animal responded on the sucrose spout in response to a test stimulus. Only trials with a response were used in these calculations. This Sucrose Generalization Score served to express the data in terms of the degree to which a test stimulus shared taste qualities with sucrose or quinine, while also controlling for individual differences in overall performance. A Sucrose Generalization Score of 0.0 indicates that the animal treated the test stimulus as if it were identical to $1.0 \mathrm{~mm}$ quinine, whereas a Sucrose Generalization Score of 1.0 indicates that it treated the test stimulus as if it were identical to $600 \mathrm{~mm}$ sucrose; a Sucrose Generalization Score of 0.5 could indicate that the stimulus shared no perceptual qualities with either sucrose or quinine, or it could indicate that the stimulus has perceptual qualities of both sucrose and quinine. We chose to use the performance on the highest concentrations of the standard stimuli to represent optimally the expected performance for a sucrose-like or quinine-like taste quality.

Three-way and two-way mixed ANOVAs were used to analyze performance to the standards as well as Sucrose Generalization Scores for sucralose and sucrose/quinine mixtures. Because we were testing a directional hypothesis, specifically that SA would have lower Sucrose Generalization Scores relative to SP, we conducted one-tailed independent samples $t$ tests at each concentration of sucralose. Sucrose Generalizations Scores for the water and electrolyte test stimuli were analyzed for group differences using independent $t$ tests and were then analyzed for differences from 0.0 with one-sample $t$ tests. The overall percentage correct for each water control test for each rat was tested for positive deviations above chance (i.e., 50\%) in a one-tailed normal approximation of the binomial distribution. Differences were considered statistically significant at $p \leq 0.05$.

\section{Experiment 2 \\ Subjects}

The same nine SA and SP adult male rats from Experiment 1 were tested in Experiment 2. All rats remained individually housed in polycarbonate cages in the same humidity- and temperature-controlled room on the same light cycle. Before training and throughout the first testing phase, all rats were maintained on a $23 \mathrm{~h}$ water-deprivation schedule in which rats received $1 \mathrm{~h}$ water access daily at the cessation of the training and testing sessions. All rats had ad libitum access to pelleted rat chow (Purina 5001) in their home cage.

\section{Stimuli}

All test solutions were made daily with RO water and reagent grade chemicals (Tate and Lyle) and presented at room temperature. Testing solutions consisted of the same nine concentrations $(0.0025-80 \mathrm{~mm})$ of sucralose that were used in Experiment 1 and water.

\section{Apparatus}

We examined the unconditioned affective licking response of each animal using a commercially purchased lickometer called a Davis rig (MS80; DiLog Instruments). The Davis rig consists of a polycarbonate cage with a wire mesh floor and a centrally located opening that is occluded by a computer-controlled shutter (Smith, 2001). A computer-controlled sliding table, with 16 fluid reservoirs connected to sipper tubes coupled to a contact lickometer, is positioned on the other side of the shutter. This device allows each animal brief access to a single drinking spout through the centrally positioned slot in the front wall of the cage when the shutter is open. Lick contact is measured by a specially designed high-frequency AC electrical circuit that minimizes electrical stimulation of the tongue and is recorded in the software control program for later analysis.

\section{Training}

On the first day of training, water-deprived rats were individually placed in the Davis rig and allowed to drink water ad libitum from a stationary fluid reservoir while the shutter remained opened for a period of $10 \mathrm{~min}$. The following day, each rat was individually placed in the Davis rig while a computer-operated protocol was run. Ten fluid reservoirs were filled with $\mathrm{RO}$ water and rats were given access to one reservoir at a time for $1 \mathrm{~min}$; upon initiation of a lick, the reservoir was presented for $30 \mathrm{~s}$ followed by a $10 \mathrm{~s}$ interpresentation interval. Two randomized blocks of all 10 fluid reservoirs were presented, giving a total of 20 presentations per rat.

\section{Testing}

The same protocol used on the second day of training was adopted for sucralose testing sessions with the exception that nine of the 10 reservoirs were filled with the various concentrations of sucralose solutions. Once the animals had completed testing in a water-deprived state, the same experiment (without the two training days) was repeated with the animals in a need-free state. Two randomized blocks of 10 solutions (RO water and nine concentrations of sucralose) were presented per day. Rats were tested for $5 \mathrm{~d}$ per each physiological state. One animal died before completing the testing sessions and its data were discarded from this analysis. Following completion of the second experiment, animals received a series of $24 \mathrm{~h}$ two-bottle sucralose preference tests across a range of concentrations $(0.0025,0.025,0.25,2.5 \mathrm{mM})$ presented in an ascending series to confirm that each animal's original classification as an SA or SP remained unchanged (data not shown). Each concentration was available for $48 \mathrm{~h}$ and the bottle positions were switched at each $24 \mathrm{~h}$ interval.

\section{Data analysis}

Data from water-deprived rats are presented as a taste/water ratio representing the number of licks across all five testing days to both presentations of each concentration of sucralose relative to the number of licks across those same days of RO water (Glendinning et al., 2002; Grobe and Spector, 2008). Specifically, the mean number of licks of a given concentration of sucralose was divided by the mean number of licks of water. Data from the need-free phase of the experiment are presented as a lick score. Specifically, the average number of licks of water was subtracted from the average number of licks of a given concentration of sucralose. As we were specifically interested in the orosensory-driven response to sucralose, trials for which an animal did not sample from the presented reservoir were not included in the data analysis. In both testing conditions, all concentrations were sampled at least once by every rat. Twoway mixed ANOVAs were conducted to analyze the taste/water ratios and lick scores. Similar to Experiment 1, we were specifically testing a directional hypothesis and therefore conducted one-tailed independent samples $t$ tests at each concentration of sucralose to identify at which concentrations the scores of SA and SP rats differed. Differences were considered statistically significant at $p \leq 0.05$.

\section{Results \\ Experiment 1}

Testing: standard stimuli and sucralose

Two-way ANOVAs for each standard stimulus indicated no overall group differences in performance on the identification of either sucrose $\left(F_{(1,7)}=1.23, p=0.31\right)$ or quinine $\left(F_{(1,7)}=0.37, p=0.56\right)$ across all of the testing sessions (Table 1 ). There was also no significant interaction between group and concentration (sucrose: $F_{(2,14)}=1.19, p=0.33$; quinine: $\left.F_{(2,14)}=0.002, p=0.99\right)$. There 
Table 1. Mean ( \pm SEM) percentage of correct responses of SP and SA rats for the standard stimuli across testing sessions

\begin{tabular}{|c|c|c|c|c|c|c|}
\hline \multirow[b]{3}{*}{ Phenotype } & \multicolumn{6}{|l|}{ Stimulus } \\
\hline & \multicolumn{3}{|l|}{ Sucrose } & \multicolumn{3}{|l|}{ Quinine } \\
\hline & $60 \mathrm{~mm}$ & $200 \mathrm{~mm}$ & $600 \mathrm{~mm}$ & $0.1 \mathrm{~mm}$ & $0.3 \mathrm{~mm}$ & $1.0 \mathrm{~mm}$ \\
\hline SP & $0.64 \pm 0.07$ & $0.94 \pm 0.01$ & $0.96 \pm 0.02$ & $0.90 \pm 0.02$ & $0.93 \pm 0.02$ & $0.94 \pm 0.01$ \\
\hline SA & $0.73 \pm 0.04$ & $0.96 \pm 0.01$ & $0.97 \pm 0.01$ & $0.92 \pm 0.03$ & $0.94 \pm 0.01$ & $0.96 \pm 0.01$ \\
\hline
\end{tabular}

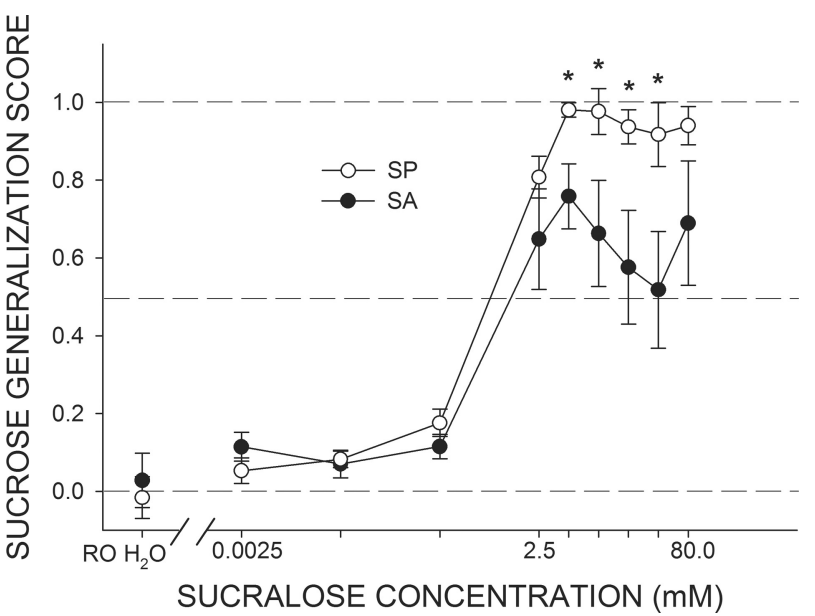

Figure 1. Mean ( \pm SEM) Sucrose Generalization Scores for SA and SP rats in response to increasing concentrations of sucralose used as a test stimulus. A score of 0.0 indicates that responding to the test stimulus was identical to responding for $1.0 \mathrm{~mm}$ quinine; a score of 1.0 indicates that responding to the test stimulus was identical to responding for $600 \mathrm{~mm}$ sucrose. All rats, regardless of sucralose preference phenotype, treated water and the three lowest concentrations of sucralose as sharing a perceptual quality similar to that of $1.0 \mathrm{~mm}$ quinine. As the concentration of sucralose was increased beyond $2.5 \mathrm{~mm}$, mean responding by SP and SA rats began to separate, with SP rats reporting that the salient taste quality associated with sucralose was similar to that of $600 \mathrm{~mm}$ sucrose, whereas SA displayed a pattern of responses indicative of sucralose sharing perceptual qualities of both $600 \mathrm{~mm}$ sucrose and $1.0 \mathrm{~mm}$ quinine. *SP rats greater than $S A$ rats, $p s<0.05$.

was, however, a significant main effect of concentration for performance (sucrose: $F_{(2,14)}=56.75, p<0.001$; quinine: $F_{(2,14)}=11.87$, $p<0.002)$. Although the rats displayed concentration-dependent performance, overall performance across the standard stimuli was quite high, indicating that the rats had competently learned the task.

The mean Sucrose Generalization Scores for SA and SP rats are depicted in Figure 1. At the three lowest concentrations of sucralose $(0.0025-0.25 \mathrm{mM})$, both groups treated sucralose as if it primarily elicited a quinine-like taste quality. Starting at the $2.5 \mathrm{~mm}$ concentration, both SA and SP rats began to report that sucralose contained a sucrose-like quality. A two-way ANOVA of Sucrose Generalization Scores revealed that the main effect of group just missed statistical significance $\left(F_{(1,7)}=4.73, p=0.06\right)$. However, we found an interaction between group and concentration $\left(F_{(8,56)}=2.17, p<0.05\right)$. SP rats monotonically increased their Sucrose Generalization Scores as sucralose concentration was raised, apparently reaching an asymptote at the $5.0 \mathrm{~mm}$ concentration. In contrast, the Sucrose Generalization Scores of the SA rats peaked at $5.0 \mathrm{~mm}$ and then started to reverse direction, indicating that as the concentration was raised, sucralose started to taste less like sucrose, except, perhaps, for the very highest sucralose concentration. Indeed, at all of the sucralose concentrations $>2.5 \mathrm{~mm}$ except at the highest, the Sucrose Generalization Scores for SA rats were lower than those for SP rats (all ps $\leq$ 0.05). The Sucrose Generalization Scores of SA rats tended to increase again at the highest sucralose concentration tested $(80 \mathrm{~mm})$.

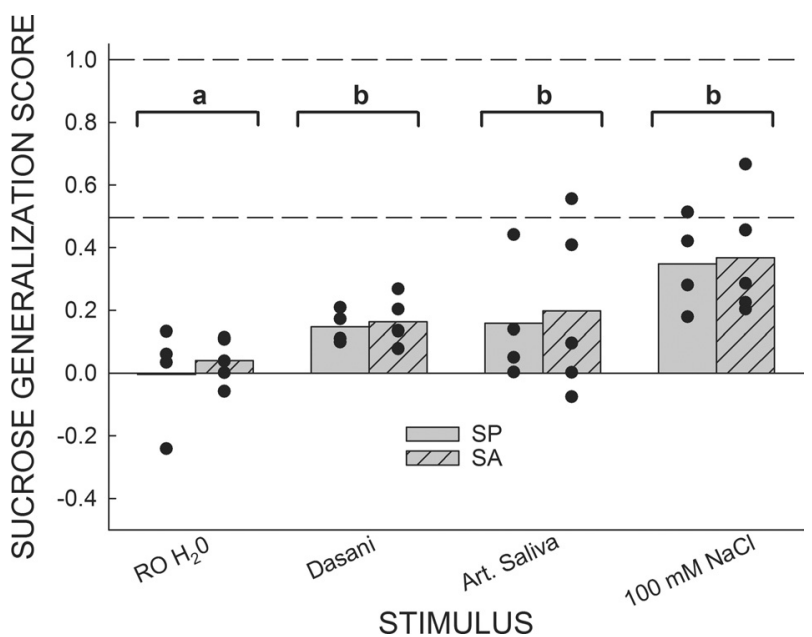

Figure 2. Mean Sucrose Generalization Scores for SA and SP rats in response to RO water and a number of electrolyte-containing stimuli. Filled circles denote the Sucrose Generalization Score for each individual rat. A score of 0.0 indicates that responding to the test stimulus was identical to responding for $1.0 \mathrm{~mm}$ quinine; a score of 1.0 indicates that responding to the test stimulus was identical to responding for $600 \mathrm{~mm}$ sucrose. No differences between SP and SA rats were detected in response to any stimulus; as such, the data were collapsed across groups for further analyses (indicated by brackets). As the amount of dissolved electrolytes was increased, the mean Sucrose Generalization Scores displayed a tendency to approach a score of 0.5 . Onesample $t$ tests revealed that $\mathrm{RO}_{2} \mathrm{O}$ was not treated as significantly different from $1.0 \mathrm{~mm}$ quinine (bracket a), while Dasani bottled water, artificial (Art.) saliva, and $100 \mathrm{~mm} \mathrm{NaCl}$ ) were treated as significantly different from $1.0 \mathrm{~mm}$ quinine ( $p s \leq 0.05$; bracket $b$ ).

Water and electrolyte test sessions

Due to the three lowest concentrations of sucralose (0.0025-0.25 $\mathrm{mm}$ ) being reported by both groups as decidedly quinine-like, we ran a series of tests to determine how rats, trained using this paradigm, would generalize $\mathrm{RO}$ water (used as solvent and reinforcer), Dasani bottled water, artificial saliva, and $100 \mathrm{mM} \mathrm{NaCl}$ (Fig. 2). No group differences were detected for each of the four test stimuli ( $p s>0.6)$; thus, the data were collapsed across groups and one-sample $t$ tests comparing the Sucrose Generalization Scores to 0.0 , a value indicating that the test stimulus was treated as qualitatively identical to $1.0 \mathrm{~mm}$ quinine, were conducted. The rats treated $\mathrm{RO}$ water as if it were not significantly different from $1.0 \mathrm{~mm}$ quinine $(p=0.6)$. Dasani bottled water, artificial saliva, and $100 \mathrm{~mm} \mathrm{NaCl}$ were all treated as being significantly different from $1.0 \mathrm{~mm}$ quinine $(p s \leq 0.05)$.

\section{Sucrose/quinine mixture test sessions}

We examined the sucrose and quinine mixture data (Table 2) in a three-way mixed ANOVA and found significant effects of sucrose concentration $\left(F_{(3,21)}=41.90, p<0.001\right)$, quinine concentration $\left(F_{(3,21)}=10.39, p<0.001\right)$, and an interaction between the sucrose and quinine concentrations $\left(F_{(9,63)}=15.92, p<0.001\right)$. There was no main effect of group $\left(F_{(1,7)}=0.04, p=0.9\right)$, no two-way interactions involving group by quinine concentration $\left(F_{(3,21)}=0.52, p=0.7\right)$ or group by sucrose concentration $\left(F_{(3,21)}=0.18, p=0.9\right)$, and no three-way interaction 
Table 2. Mean ( \pm SEM) Sucrose Generalization Scores ${ }^{a}$ of SP and SA rats for sucrose/quinine taste mixtures

\begin{tabular}{|c|c|c|c|c|c|c|c|c|}
\hline \multirow[b]{3}{*}{ Quinine (mм) } & \multicolumn{8}{|l|}{ Sucrose (mм) } \\
\hline & \multicolumn{2}{|l|}{75} & \multicolumn{2}{|l|}{150} & \multicolumn{2}{|l|}{300} & \multicolumn{2}{|l|}{600} \\
\hline & $S P$ & SA & $S P$ & SA & $S P$ & SA & $S P$ & $S A$ \\
\hline 0.3 & $0.68 \pm 0.11$ & $0.80 \pm 0.04$ & $0.97 \pm 0.04$ & $0.91 \pm 0.04$ & $0.95 \pm 0.03$ & $0.98 \pm 0.02$ & $0.93 \pm 0.05$ & $1.0 \pm 0.02$ \\
\hline 1.0 & $0.60 \pm 0.06$ & $0.60 \pm 0.09$ & $0.63 \pm 0.23$ & $0.76 \pm 0.06$ & $1.0 \pm 0.01$ & $1.0 \pm 0.04$ & $1.2 \pm 0.05$ & $1.0 \pm 0.04$ \\
\hline 3.0 & $0.29 \pm 0.13$ & $0.16 \pm 0.07$ & $0.86 \pm 0.16$ & $0.83 \pm 0.14$ & $0.87 \pm 0.02$ & $0.77 \pm 0.10$ & $0.91 \pm 0.06$ & $0.88 \pm 0.09$ \\
\hline
\end{tabular}

${ }^{a} \mathrm{~A}$ score of 0.0 indicates that responding to the test stimulus was identical to responding for $1.0 \mathrm{~mm}$ quinine; a score of 1.0 indicates that responding to the test stimulus was identical to responding for $600 \mathrm{~mm}$ sucrose.

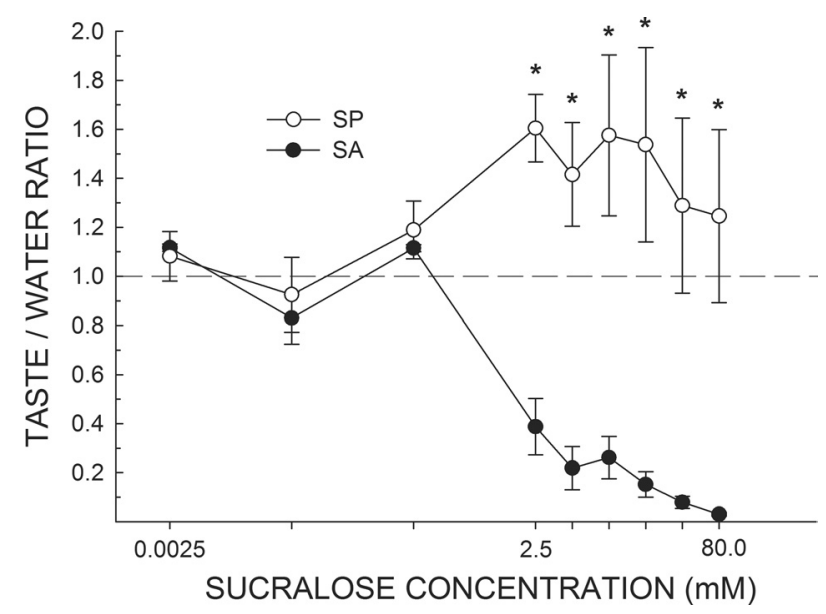

Figure 3. Mean ( \pm SEM) taste/water ratio of water-deprived SA and SP rats in response to increasing concentrations of sucralose presented in random order during brief-access tests in the Davis rig. A score of 1.0 indicates that the mean licking response to that concentration of sucralose was equal to the overall mean licking response to water. In agreement with Experiment 1, all rats treated the three lowest concentrations of sucralose as similar to water. By the $2.5 \mathrm{~mm}$ concentration, SP rats increased licking and SA rats decreased licking in response to increasing concentrations of sucralose. ${ }^{*} S P$ rats greater than $S A$ rats, $p s \leq 0.01$.

$\left(F_{(9,63)}=1.86, p=0.08\right)$. Furthermore, separate two-way ANOVAs conducted for each base concentration of sucrose revealed that rats in both groups displayed increasing responses on the quinine reinforcement spout as the concentration of quinine was raised, as indicated by the mean decrease in Sucrose Generalization Scores $\left(75 \mathrm{~mm}: F_{(3,21)}=41.65, p<\right.$ $0.001 ; 150 \mathrm{~mm}: F_{(3,21)}=3.54, p<0.05 ; 300 \mathrm{mM}: F_{(3,21)}=5.25$, $p<0.01$; and $\left.600 \mathrm{~mm}: F_{(3,21)}=7.32, p<0.01\right)$.

\section{Water control sessions}

As a negative control, we examined performance of both groups when presented with only $\mathrm{RO}$ water with some reservoirs arbitrarily assigned as sucrose and some assigned as quinine. Every rat performed at chance levels when all reservoirs were filled with RO water (range: $45.0-53.1 \%$ ). Interestingly, the tendency for the rats to report water as similar to quinine basically disappeared on average in this test in which the three sucrose and quinine standards were not presented; the percentage of "correct" responses when presented with fluid from one of the three arbitrarily assigned quinine reservoirs was $50.1 \pm 13.5 \%$ and $47.0 \pm 12.5 \%$ for SP and SA rats, respectively. This strongly suggests that the seemingly bitter taste of water may be dependent on the presence of a comparative stimulus and could be due to an interactive effect of sucrose and/or quinine during the test sessions.

\section{Experiment 2}

When assessed in the brief-access test while water-deprived, the same SA rats from Experiment 1 displayed a very different

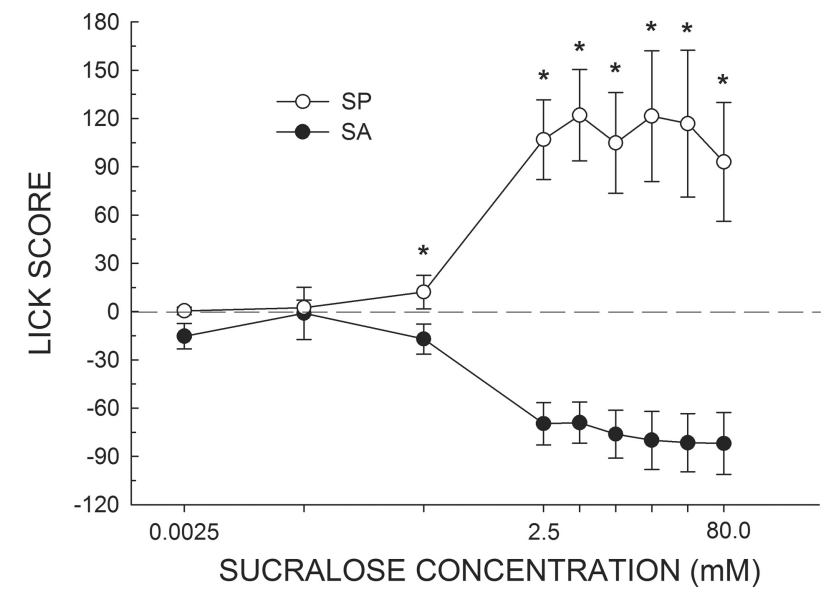

Figure 4. Mean ( \pm SEM) lick score of need-free SA and SP rats in response to increasing concentrations of sucralose presented in random order during brief-access tests in the Davis rig. Scores $>0.0$ indicate that the animals licked more to sucralose relative to water; scores $<0.0$ indicate that the animals licked more to water relative to sucralose. Comparable to Figure 3, SA and SP rats treated the two lowest concentrations of sucralose as similar to water. Interestingly, in a water-replete state, SA and SP rats displayed reliable differences in their licking response at a $1 \log$ unit lower concentration of sucralose $(0.25 \mathrm{~mm})$ compared with the fluid-deprived state $(2.5 \mathrm{~mm}) .{ }^{*} \mathrm{SP}$ rats greater than $\mathrm{SA}$ rats, $p s<0.05$.

concentration-dependent profile of licking compared with SP rats (Fig. 3). SA rats generally decreased their licking as a function of sucralose concentration. In contrast, SP rats increased their licking as sucralose concentration was raised, reaching a plateau beginning at the $2.5 \mathrm{~mm}$ concentration. A two-way ANOVA revealed an interaction between group and concentration for taste/ water ratios $\left(F_{(8,48)}=12.78, p<0.001\right)$. Importantly, the mean taste/water ratio of SP rats never dropped below 0.93 , indicating that the presumably thirsty SP rats were licking sucralose almost as much or more than they were water. In contrast, the mean taste/water ratio for SA rats, while close to 1.0 for the three lowest sucralose concentrations, as was true for the SP rats, precipitously dropped by the $2.5 \mathrm{~mm}$ concentration. The SA rats continued to decrease their licking as the concentration was raised until reaching a taste/water ratio close to $0(0.02 \pm 0.01)$. The mean taste/ water ratios of SA and SP rats significantly differed at every concentration $\geq 2.5 \mathrm{~mm}(p s \leq 0.01)$.

When the rats were tested in the brief-access test in a need-free state, there was a significant difference between the groups in how they responded to water $\left(t_{(6)}=2.9, p<0.03\right)$. We therefore subtracted the average water licking response for each rat from its average licking response to each concentration of sucralose to form lick scores (Fig. 4). A two-way ANOVA of the lick scores revealed a group by concentration interaction $\left(F_{(8,48)}=14.92\right.$ $p<0.001)$. When tested in a need-free state, the lick scores of SA and SP rats first diverged at a lower concentration $(0.25 \mathrm{~mm})$ compared with when the animals were tested in a water-deprived 
physiological state $(p<0.05)$. At every concentration $>0.25 \mathrm{~mm}$, the mean lick scores of SA and SP rats significantly differed ( $p s<$ $0.01)$. At no point did SA rats lick more to sucralose than to water.

\section{Discussion}

The results from these experiments suggest that the mechanism responsible for the phenotypic difference in preference for and affective responsiveness to sucralose in rats has a sensory origin. Rats that were highly trained to discriminate between the taste quality of sucrose and quinine treated high concentrations of sucralose as sucrose-like significantly more so if they were categorized as SP rather than SA. The response patterns in the sensory discrimination task and the affective-driven licking tasks corresponded closely. Accordingly, it appears that the taste quality perceived by SP rats differs from that perceived by SA rats and thus it is likely that the sensory signal provided to reward circuits is not the same for these two phenotypic classes of animals.

Both SA and SP rats treated RO water and the three lowest concentrations of sucralose as quinine-like, rather than sucroselike. Together, these findings suggest that with the small volumes used in the operant discrimination task, the low concentrations of sucralose were close to or below the detection threshold and thus were treated as water. Similarly, in the brief-access tests, SA and SP rats treated these three lowest sucralose concentrations as they did water in both a water-deprived and need-free state, further suggesting these concentrations are weak.

It is unclear why $\mathrm{RO}$ water, within this testing paradigm, possesses a mild quinine-like taste, but others have also found that rats and humans treat water as possessing a bitter taste quality under certain test conditions (Morrison, 1967; Bartoshuk, 1977; Grobe and Spector, 2008). It has previously been speculated that the bitter taste of water may be due to the inherent rinsing away of various compounds, including those present in saliva, adapting the taste receptor cells and thus represents a gustatory analog to visual afterimages (Galindo-Cuspinera and Breslin, 2007). It seems likely that the presentation of the many sucrose and quinine concentrations during the session created a very specific taste receptor adaptation background, despite the many water rinses that occurred upon reinforcer delivery, and that led to RO water, as well as the low concentrations of sucralose, bottled water, artificial saliva, and, to some extent, $100 \mathrm{~mm} \mathrm{NaCl}$, being reported as bitter. Support for such a possible interaction comes from the water control test in which only RO water was presented during the session. When we examined the responses on quinineversus sucrose-simulated trials, there was no consistent bias for rats to treat $\mathrm{RO}$ water as quinine-like.

SA and SP rats first displayed an increase in responding on the sucrose reinforcement spout at $2.5 \mathrm{~mm}$ sucralose, indicating that the salient taste quality was predominately sucrose-like. SP rats continued to treat higher concentrations of sucralose as if they elicited solely a sucrose-like quality, whereas SA rats were less likely to respond on the sucrose spout than the SP rats as the concentration of sucralose increased up to $40 \mathrm{~mm}$. As such, the differential responding between the groups was most pronounced at $40 \mathrm{~mm}$ sucralose, at which concentration SP and SA rats displayed mean Sucrose Generalization Scores of 0.91 and 0.51 , respectively. On average, the SA group never reached a Sucrose Generalization Score $<0.5$, indicating an approximately equal number of responses on the sucrose and quinine reinforcement spouts. Although this finding could suggest that high concentrations of sucralose generate a taste quality that is distinct from both sucrose and quinine to SA rats, a more parsimonious interpretation of this response pattern is that SA rats perceived the taste quality of sucralose as similar to both sucrose and quinine, an expected result if sucralose, in fact, elicited a bittersweet taste quality, as is commonly reported for artificial sweeteners in human subjects (Schiffman et al., 1995; Horne et al., 2002; Roudnitzky et al., 2011). Increasing the concentration would presumably increase the contribution of both of these qualitative components simultaneously. Thus, it should not necessarily be expected that increasing the concentration of sucralose should render the stimulus completely like quinine to SA rats. Both the preference behavior and the unconditioned licking responses in the brief-access tests indicate that the quinine-like signal is, however, sufficiently aversive to compete with the positive motivational potency of the sucrose-like signal at the higher sucralose concentrations in SA rats.

All rats increased responding on the quinine reinforcement spout as the concentration of quinine adulterating all of the sucrose base solutions was increased. However, the Sucrose Generalization Score only dropped below 0.5 at the least concentrated sucrose base solution. Binary bitter-sweet mixtures exhibit a mutually, albeit unequal, suppressive effect on the intensity of the two taste qualities (Bartoshuk, 1975; Lawless, 1982; Formaker et al., 1997). Likewise, Kroeze and Bartoshuk (1985) demonstrated that when sucrose and quinine are applied to different, nonoverlapping portions of the tongue, subjects report that the quinine contribution is suppressed relative to the sucrose. Thus, a central mechanism is likely involved in taste mixture suppression. In human studies, sucrose appears to be the least suppressed and the strongest suppressor of other tastes (Green et al., 2010); studies in rodents are limited and more equivocal (Formaker et al., 1997). Mixture suppression may explain why the sucrose quality appeared to be the more salient taste quality until the concentration was sufficiently lowered ( $75 \mathrm{~mm}$ ) even in the face of relatively intense quinine adulteration. Nevertheless, regardless of the mechanism, it is clear that there was a strong bias toward treating mixtures of sucrose and quinine as predominantly sucrose-like in this task. Even though there was not a difference between the two groups in their generalization profiles to the sucrose/quinine mixtures, the tendency to report that the mixtures were sucroselike might explain why the average sucrose generalization scores for high concentrations of sucralose did not drop below 0.5 in the SA group.

Although we systematically varied the component concentrations spanning a relatively wide range, no group differences were observed in the Sucrose Generalization Scores associated with any of the sucrose/quinine mixtures. This suggests that the phenotypic split in the Sucrose Generalization Scores associated with sucralose is specific to this artificial sweetener and may not affect all putatively sweet- and bitter-tasting stimuli. It would be instructive to investigate other artificial sweeteners to examine whether SA and SP rats would treat them as they do sucralose in this discrimination task. For example, rats have been selectively bred for high or low saccharin preference in two-bottle tests (for review, see Carroll et al., 2008). Interestingly, these saccharin preference phenotypes correspond with responses to other rewarding stimuli. To our knowledge, such animals have not been tested for their sensory function. If indeed the SA and SP phenotypes are specific to sucralose, then this would point to a peripheral origin of the difference, possibly involving a mutation in one or more T2Rs, the family of taste receptors that bind with bitter-tasting ligands (Chandrashekar et al., 2000), activated by sucralose.

Although we cannot conclusively rule out that stimulus palatability may have served as a discriminative cue, the profile of 
results suggest otherwise. First, although the palatability of sucrose and quinine are clearly concentration-dependent, the responses across the concentrations were relatively stable except for the weakest sucrose stimulus, for which performance modestly dropped. Second, the palatability of water differs markedly from quinine, especially to a water-deprived rat, yet all animals treated water as if it was similar to $1.0 \mathrm{~mm}$ quinine-a finding, as noted above, that is supported by other psychophysical studies involving rats and humans. This does not mean that water has an intensely bitter taste; it merely means that whatever weak taste it generates under these test conditions, its quality is much more similar to quinine than to sucrose when both are presented concurrently within the test. Third, although in our measures of sucralose palatability in the brief-access test, SA rats strongly avoided sucralose and SP rats avidly licked the higher sucralose concentrations, the mean Sucrose Generalization Scores of SA rats tested at the same concentrations in the discrimination task never dropped below 0.5 . This strongly suggests that responses were not being guided by the palatability of the taste stimulus. Rather, the most parsimonious interpretation of this outcome is that, at higher concentrations, sucralose has both a sweet and bitter taste and the difference between the behavior of the SP and SA rats in the generalization test is related to the relative contributions of the two qualitative components.

Neural structures implicated in reward processing receive their input from sensory systems. Thus, a given anatomical, genetic, or pharmacological manipulation that affects the apparent reward value of a stimulus (Cromwell and Berridge, 1993; Doyle et al., 1993; Kelley et al., 2002; Tindell et al., 2006) could be acting on sensory processes, reward processes, or both. It is impossible to distinguish decisively among these possibilities without an explicit test of the effect of a given manipulation on sensory function independent of the rewarding characteristics of the stimulus. Many studies of the neural mechanisms of reward and affect use taste stimuli (Roitman et al., 2005). Sucrose and artificial sweeteners are especially popular in this regard (Geary and Smith, 1985; Yamamoto, 2003; Hajnal et al., 2004). The present study both provides an example of how a psychophysical analysis could help disambiguate the interpretation of interventions that affect the reward value of, or preference for, taste stimuli and demonstrates the importance of considering alterations in sensory processes as the cause of the effect.

\section{References}

Bartoshuk LM (1975) Taste mixtures: is mixture suppression related to compression? Physiol Behav 14:643-649.

Bartoshuk LM (1977) Water taste in mammals. In: Drinking behavior (Weijnen J, Mendelson J, eds.), pp 317-339. New York: Plenium.

Carroll ME, Morgan AD, Anker JJ, Perry JL, Dess NK (2008) Selective breeding for differential saccharin intake as an animal model of drug abuse. Behav Pharmacol 19:435-460.

Chandrashekar J, Mueller KL, Hoon MA, Adler E, Feng L, Guo W, Zuker CS, Ryba NJ (2000) T2Rs function as bitter taste receptors. Cell 100: 703-711.

Cromwell HC, Berridge KC (1993) Where does damage lead to enhanced food aversion: the ventral pallidum/substantia innominate or lateral hypothalamus? Brain Res 624:1-10.

Doyle TG, Berridge KC, Gosnell BA (1993) Morphine enhances hedonic taste in palatability in rats. Pharmacol Biochem Behav 46:745-749.

Formaker BK, MacKinnon BI, Hettinger TP, Frank ME (1997) Opponent effects of quinine and sucrose on single fiber taste responses of the chorda tympani nerve. Brain Res 772:239-242.

Galindo-Cuspinera V, Breslin PA (2007) Taste after-images: the science of "water-tastes". Cell Mol Life Sci 64:2049-2052.

Geary N, Smith GP (1985) Pimozide decreases the positive reinforcing effect of sham fed sucrose in the rat. Pharmacol Biochem Behav 22:787-790.

Glendinning JI, Gresack J, Spector AC (2002) A high-throughput screening procedure for mice with aberrant taste and oromotor function. Chem Senses 27:461-474.

Green BG, Lim J, Osterhoff F, Blacher K, Nachtigal D (2010) Taste mixture interactions: suppression, additivity, and the predominance of sweetness. Physiol Behav 101:731-737.

Grobe CL, Spector AC (2008) Constructing quality profiles for taste compounds in rats: a novel paradigm. Physiol Behav 95:413-424.

Hajnal A, Smith GP, Norgren R (2004) Oral sucrose stimulation increases accumbens dopamine in the rat. Am J Physiol Regul Integr Comp Physiol 286:R31-R37.

Horne J, Lawless HT, Speirs W, Sposato D (2002) Bitter taste of saccharin and acesulfame-K. Chem Senses 27:31-38.

Inoue M, Glendinning JI, Theodorides ML, Harkness S, Li X, Bosak N, Beauchamp GK, Bachmanov AA (2007) Allelic variation of the Tas1r3 taste receptor gene selectively affects taste responses to sweeteners: evidence from 129.B6-Tas1r3 congenic mice. Physiol Genomics 32:82-94.

Kelley AE, Bakshi VP, Haber SN, Steininger TL, Will MJ, Zhang M (2002) Opioid modulation of taste hedonics in the ventral striatum. Physiol Behav 76:365-377.

Kroeze JH, Bartoshuk LM (1985) Bitterness suppression as revealed by split-tongue taste stimulation in humans. Physiol Behav 35:779-783.

Lawless H (1982) Paradoxical adaptation to taste mixtures. Physiol Behav 29:149-152.

Loney GC, Torregrossa AM, Sclafani A, Smith JC, Eckel LA (2011) Rats display a robust bimodal preference profile for sucralose. Chem Senses 36:733-745

Morrison GR (1967) Behavioral response patterns to salt stimuli in the rat. Can J Psychol 21:141-152.

Roitman MF, Wheeler RA, Carelli RM (2005) Nucleus accumbens neurons are innately tuned for rewarding and aversive taste stimuli, encode their predictors, and are linked to motor output. Neuron 45:587-597.

Roudnitzky N, Bufe B, Thalmann S, Kuhn C, Gunn HC, Xing C, Crider BP, Behrens M, Meyerhoff W, Wooding SP (2011) Genomic, genetic and functional dissection of bitter taste responses to artificial sweeteners. Hum Mol Genet 20:3437-3449.

Schiffman SS, Booth BJ, Losee ML, Pecore SD, Warwick ZS (1995) Bitterness of sweeteners as a function of concentration. Brain Res Bull 36:505-513.

Sclafani A, Clare RA (2004) Female rats show a bimodal preference response to the artificial sweetener sucralose. Chem Senses 29:523-528.

Smith JC (2001) The history of the Davis rig. Appetite 36:93-98.

Spector AC, Glendinning JI (2009) Linking peripheral taste processes to behavior. Curr Opin Neurobiol 19:370-377.

Spector AC, Andrews-Labenski J, Letterio FC (1990) A new gustometer for psychophysical taste testing in the rat. Physiol Behav 47:795-803.

Tindell AJ, Smith KS, Peciña S, Berridge KC, Aldridge JW (2006) Ventrial pallidum firing codes hedonic reward: when a bad taste turns good. J Neurophysiol 96:2399-2409.

Yamamoto T (2003) Brain mechanisms of sweetness and palatability. Nutr Rev 61:S5-S9. 